\title{
Le modèle HFO (haute fiabilité organisationnelle) : un équilibre entre les hommes, l'organisation, les outils et les situations
}

\author{
HRO (high reliability organization) model: a balance between man, organization, tools \\ and situations
}

\section{T. Desmettre}

Reçu le 30 novembre 2013; accepté le 2 décembre 2013

(C) SFMU et Springer-Verlag France 2013

C'est en 1979 qu'ont été créés en France les centres de réception et de régulation des appels (CRRA) dont l'objectif initial était de limiter les sorties des Smur... que l'on venait juste de mettre en place. Cet objectif s'est considérablement modifié et la régulation médicale occupe en effet désormais une place centrale et croissante : elle est devenue progressivement le pivot de l'organisation et du fonctionnement de l'aide médicale urgente, de la permanence des soins en médecine ambulatoire, mais également un élément central de la gestion des crises sanitaires. L'évolution de ces rôles et missions n'est pas sans conséquences. D'une part la progression constante des appels nécessite qu'une évaluation et un tri efficace soient réalisés dès la prise d'appel, que les ratios en personnels soient adaptés aux pics d'activité ; l'obtention de cette adéquation n'est pas toujours simple dans le contexte contraint du cadre hospitalier de la fonction publique. D'autre part, la nécessité d'anticipation et le concept médical de gravité potentielle conduisent à un changement de paradigme, avec l'application d'une stratégie de bénéfice du doute, au profit du patient [1,2]. L'application de cette stratégie fait écho au concept juridique de perte de chance : plus l'état de santé du patient est alarmant et plus la localisation géographique est distante d'un établissement de santé pouvant le prendre en charge, plus il a de risque de subir un préjudice. Enfin l'environnement technologique s'est modifié, il doit continuer d'évoluer et de se moderniser avec l'implantation de nouveaux outils, dont le corollaire est la complexification des systèmes, inéluctable afin de répondre à l'augmentation de l'activité.

T. Desmettre $(\bowtie)$

Service d'accueil des urgences/Samu 25, CHRU de Besançon,

1, boulevar d Fleming, F-25030 Besançon cedex, France

e-mail : tdesmettre@chu-besancon.fr

Université de Franche Comté, 1, rue Claude Goudimel,

F-25030 Besançon cedex, France
Coïncidence ..., c'est également en 1979 que survenait aux États-Unis l'accident à la centrale nucléaire de Three Mile Island en Pennsylvanie, provoqué par une série de défaillances matérielles et humaines conduisant à la fusion partielle du cœur du réacteur nucléaire. Le choc provoqué par cet accident a été considérable et de nombreux enseignements en ont été tirés, notamment en France. À la suite de cet accident, des travaux ont montré qu'on ne peut aborder la sécurité sous le seul angle des "opérateurs de première ligne ", mais qu'il faut élargir l'analyse au fonctionnement organisationnel du système. Des premiers travaux ont cherché à comprendre pourquoi les systèmes complexes étaient générateurs d'accidents, puis un courant de recherche regroupé sous le nom HRO s'est intéressé aux raisons conduisant certaines organisations à être ultra-fiables. Les High Reliability Organizations, en français HFO (haute fiabilité organisationnelle), représentent une discipline de gestion des risques qui aborde la sécurité sous l'angle de la fiabilité organisationnelle. Les racines du paradigme HRO ont été élaborées par un groupe de chercheurs de l'Université de Californie à Berkeley qui a examiné les porte-avions, le système du contrôle du trafic aérien, des centrales nucléaires, mais également le système de commandement des interventions de feu, de l'Unité de soins intensifs pédiatriques de Loma Linda Hospital... Même si elles semblent très différentes à première vue, ces organisations présentent un certain nombre de similitudes. Tout d'abord, elles opèrent dans des environnements sociaux et politiques omniprésents et qualifiés d' « impitoyables ». Deuxièmement, leurs technologies présentent un risque d'erreur. Troisièmement, l'ampleur des conséquences possibles d'erreurs ou de fautes empêche l'apprentissage par l'expérimentation. Enfin, pour éviter les échecs, ces organisations utilisent des processus complexes pour gérer des technologies... complexes, et un travail... complexe. Les travaux de Weick et Sutcliffe de 2001 sur les organisations nécessitant une grande fiabilité mettent en avant cinq processus clés permettant de maintenir, au 
quotidien, un management de l'inattendu : l'attention portée aux échecs plutôt qu'aux succès ; la méfiance envers la simplification des interprétations ; la sensibilisation aux opérations (et leur caractère délicat) ; l'obligation de résilience ; la déférence envers l'expertise, pour garantir la fluidité du système de prise de décision [3]. La combinaison de ces cinq processus permet de produire un état de «vigilance collective » (mindfulness), correspondant à une sorte d'état d'alerte permanent, grâce à la qualité des interactions au sein de l'organisation. Être attentif consiste dans ce cas à développer une conscience aiguë des détails discriminatoires ainsi qu'une capacité accrue à découvrir et à corriger les erreurs qui pourraient se transformer en crise. Dans l'analyse de l'organisation il ressort que la sécurité est une propriété qui émerge des relations, rites et mythes existant au niveau de l'individu et du groupe, mais plus encore au niveau de l'organisation tout entière. La sécurité est construite de façon à répondre aux demandes de l'environnement, et en particulier au caractère inattendu et imprévu, imprévisible de l'activité. Les organisations répondant aux critères HFO sont le fruit d'une bonne adaptation entre les demandes extérieures et la structuration des unités de l'organisation. Par conséquent, il n'existe pas une seule manière d'organiser la sécurité mais plusieurs façons peuvent exister. La vision d'une sécurité construite, contingente de l'environnement, et adaptative aux situations explique qu'elle résulte d'un équilibre circonstanciel entre les hommes, l'organisation, les outils et les situations.

L'activité des CRRA en France peut-elle être qualifiée d'activité à haut risque ? Sans doute, si l'on examine son fonctionnement en regard des critères précédemment cités. L'environnement social et politique est une composante forte, qui impacte directement son activité ; comme toutes technologies complexes, celles utilisées présentent un risque d'erreur ; enfin l'apprentissage par expérimentation est limité, compte tenu de l'ampleur des conséquences. La complexité des processus au sein des CRRA est une caractéristique qui ne fait pas débat. Dans ces conditions, analyser le fonctionnement d'un CRRA par le prisme d'une organisation HRO est intéressant. C'est l'objet du travail présenté par l'équipe du CRRA de Nantes dans ce numéro des Annales françaises de médecine d'urgence, qui confrontent leur modèle organisationnel trivalent au modèle HRO [4]. Cette approche est pertinente, même si les auteurs soulignent les limites d'une évaluation en interne. Ce travail est à mettre en perspective avec celui du rapport Mardegan, relatif à la modernisation des Samu, qui a mis à jour les fragilités opérationnelles et technologiques et proposé des pistes de réflexion [5]. À sa suite, la maîtrise d'ouvrage de la modernisation des systèmes d'information et télécoms des « Samucentres $15 »$ a été confiée à l'Agence des systèmes d'information partagés de santé (ASIP santé), dès octobre 2012 [6].
Ainsi le travail de Penverne et al. [4] apporte un éclairage et le recul indispensables à l'analyse critique de nos modes de fonctionnement. Il ouvre la porte des référentiels et du choix des critères d'évaluation, placés du point de vue des patients, de la Direction hospitalière et des professionnels du Centre 15. Les auteurs présentent ainsi leur modèle trivalent d'organisation fondé sur un système d'information performant, une gestion raisonnée des ressources humaines et un management opérationnel.

À côté des aspects techniques, certes fondamentaux, se posent ceux du facteur humain et de l'analyse qualitative du colloque singulier entre l'appelant et un professionnel non médical, assistant de régulation, et médical, le médecin régulateur du CRRA. En sus des évaluations quantitatives de la performance technique de la réponse, comme par exemple le temps de décroché, il faut développer des indicateurs de cette réponse humaine. L'évaluation de la compréhension de la demande de l'appelant, de la compréhension par l'appelant des conseils prodigués, du niveau de fiabilité des informations délivrées, comprises, transmises et retransmises, et de la distorsion de cette information sont des problématiques spécifiques. Dans ce champ de recherche, mener une réflexion multidisciplinaire avec des sociologues, psychologues, linguistes, et juristes est une voie qui mérite d'être explorée, par exemple sous l'angle large de la vulnérabilité de la réponse aux appels au « $15 »[7]$.

\section{Références}

1. Haute Autorité de la santé (HAS) (2011) Modalités de prise en charge d'un appel de demande de soins non programmés dans le cadre de la régulation médicale. www.has-sante.fr (dernier accès le 2 décembre 2013)

2. Giroud M, Pateron D (2012) Régulation médicale : la HAS édicte des recommandations sur la prise en charge d'un appel. Ann Fr Med Urg 2:4-6

3. Weick KE, Sutcliffe K (2001) Managing the unexpected : assuring high performance in an age of complexity. JosseyBass, San Francisco

4. Penverne Y, Lalande J, Labady J, et al (2014) Quelle organisation mettre en place au Samu Centre 15 pour répondre aux critères d'une organisation de haute fiabilité ? Ann Fr Med Urg 4:6-10

5. Mardegan P (2010) Rapport de la mission DGOS relative à la modernisation des Samu. État des lieux, objectifs et recommandations opérationnelles. http://www.sante.gouv.fr/IMG/pdf/mission dgos-rapport modernisation des samu-07-2010.pdf (dernier accès le 2 décembre 2013)

6. ASIP-santé (2013) Publication de la synthèse des retours de l'appel à commentaires sur l'étude de modernisation des systèmes d'information et de télécommunication des Samu et mise à jour de l'étude de modernisation. http://esante.gouv.fr/asip-sante (dernier accès le 2 décembre 2013)

7. Philippe C, Jacques-Jouvenot D, Desmettre T, et al (2011) Approche multidisciplinaire de la vulnérabilité dans le contexte de l'urgence médicale. Rev Gen Droit Med 39:157-80 